\title{
Gastric Band Removal in Revisional Bariatric Surgery: a One-Step or Two-Step Procedure?
}

\author{
K. Slim $^{1} \cdot$ B. Pereira ${ }^{2} \cdot$ J. Gagnière ${ }^{1}$
}

Published online: 28 March 2016

(C) Springer Science+Business Media New York 2016

\section{Dear Editor,}

We have read with interest the meta-analysis published by Dr Dang and colleagues [1] comparing one-step versus twostep revisional bariatric surgery with band removal aiming to answer a question having an important impact on our daily practice. The main finding was that one-step option is comparable to the two-step option. This meta-analysis deserves however some comments. There was a lack of statistically significant for leaks after sleeve gastrectomy. The value of odds ratio was 2.09 with an important confidence interval. The authors' interpretation of this result is surprisingly reassuring, while it should be interpreted with caution. We have calculated [2] that taking into account a $2 \%$ risk, the meta-analysis should include more than 900 patients (with a doubled risk for one-step, with $\alpha=5 \%$ and $\beta=20 \%$ ). In this way, the present meta-analysis is clearly underpowered and involves a type- 2 error.

On the other hand, the meta-analysis does not meet the quality standards of a good systematic review; only 15 items of the 27 ones from the PRISMA checklist [3] were reported (to cite a few: the abstract was not structured, there was no quality assessment of studies, no sensitivity analyses according to the quality of studies, no biases description, etc.).
Finally (a minor comment), we have also noticed that our work has been referenced two times (\#15 and \#17)!

Because of these several flaws, the present meta-analysis does not answer the question and does not bring us any evidence regarding the safety of one-step procedure. Hence, the present meta-analysis would not alter our daily practice.

\section{Compliance with Ethical Standards}

Conflict of Interest The authors declare that they have no competing interests.

\section{References}

1. Dang JT, Switzer NJ, Wu J, et al. Gastric band removal in revisional bariatric surgery, one-step versus two-step: a systematic review and meta-analysis. Obes Surg. 2016. doi:10.1007/s11695-016-2082-7.

2. Thorlund K, Mills EJ. Sample size and power considerations in network meta-analysis. Syst Rev. 2012;1:41.

3. Moher D, Liberati A, Tetzlaff J, et al. PRISMA group. Preferred reporting items for systematic reviews and meta-analyses: the PRISMA statement. BMJ. 2009;339:b2535.
K. Slim

kslim@chu-clermontferrand.fr

1 Department of Digestive Surgery, CHU Estaing, Clermont-Ferrand, France

2 Department of Statistics, CHU Estaing, Clermont-Ferrand, France 